\title{
Coverage, Capacity and Energy Efficiency of TV White Space Technology
}

\author{
Rodney Martinez Alonso, David Plets, \\ Margot Deruyck, Luc Martens, Wout Joseph \\ Ghent University / IMEC \\ Ghent, Belgium \\ \{rodney.martinezalonso, david.plets, margot.deruyck, \\ luc1.martens,wout.joseph\}@ugent.be
}

\author{
Glauco Guillen Nieto \\ LACETEL \\ Havana, Cuba \\ glauco@enet.cu
}

\begin{abstract}
The increasing demand of wireless telecommunication services is stressing the availability of radio spectrum to accommodate new services. Nevertheless, spectrum usage surveys performed in different regions reveal an underutilization of radio spectrum. TV White Space (TVWS) technologies allow a dynamic usage of the Very High Frequency (VHF) and Ultra High Frequency (UHF) television channels. In this paper, we investigate the coverage range, capacity, and energy efficiency of IEEE 802.22(b) technology. We consider Ghent, Belgium, to evaluate a realistic scenario. The higher coverage range is $7.9 \mathrm{~km}$ for IEEE $802.22 \mathrm{~b}$, when using Modulation and Coding Scheme (MCS) 1/2 QPSK. The best trade-off between coverage and capacity in Ghent suburban scenario is achieved for the MCS $2 / 3 \quad 16$ QAM, with an energy efficiency of 50 square $\mathbf{k m} \cdot \mathbf{M b p s} / \mathrm{W}$, a Base Station (BS) maximum delivered bitrate of $16.064 \mathrm{Mbps}$ and a coverage range of $2.9 \mathrm{~km}$. To ensure a percentage of users covered higher than $97 \%$ are required $20 \mathrm{BS}$.
\end{abstract}

Keywords- TV White Space technology; Propagation; Coverage; Energy Efficiency

\section{INTRODUCTION}

The demand for wireless communication services is increasing exponentially, stressing the capacity of wireless telecommunication networks and the availability of radio spectrum to accommodate new services [1]. Wireless communication systems are mainly based on the fixed radio spectrum resource allocation in order to avoid harmful interference among services [2]. Nevertheless, as revealed in [1] and [2] this approach leads into significant underutilization of the radio spectrum due to scattered usage across different geographical regions as well as in different periods of time.

TV White Space (TVWS) technologies allow a dynamic usage of the television channels in the Very High Frequency (VHF) and Ultra High Frequency (UHF) bands [3]. The IEEE 802.22 standard (latest update IEEE 802.22b) defines the cognitive Wireless Regional Area Network (WRAN), Medium Access Control (MAC) and Physical Layer (PHY) specifications, for TVWS systems operation in the television bands [6], [7]. IEEE 802.22(b) performs the allocation of unused spectrum by means of sensing techniques and/or a geo-location database which provides the permissible operating channels, maximum allowable transmitting power and other operating parameters [6], [7], [8].

Several trials have been conducted worldwide to evaluate the performance of TVWS technologies [9]. TV bands show excellent propagation characteristics, together with underutilization at many locations. This presents a potential spectrum-sharing opportunity [10]. In an IEEE 802.22 trial, a bit error rate of $10^{-6}$ at a distance of $6.3 \mathrm{~km}$ (one site measurement) was reported, for a MCS 3/4 64-QAM with an EIRP $=34.6 \mathrm{dBm}$, Base Station (BS) antenna height of $20 \mathrm{~m}$, receiver antenna height of $12 \mathrm{~m}$ and a receiver antenna gain of $7.65 \mathrm{dBi}$ [11].

The goal of this paper is to evaluate the coverage, capacity, and energy efficiency of IEEE 802.22 and IEEE $802.22 \mathrm{~b}$ BS in a realistic scenario, taking into consideration the required shadowing and fading margins to guarantee a certain percentage of network coverage and availability.

\section{MEthodology}

We consider Ghent, Belgium to evaluate a realistic scenario. Fig. 1 shows the target area $\left(68 \mathrm{~km}^{2}\right)$ that needs to be covered in Ghent City. The locations of the BS are represented with squares in the map.

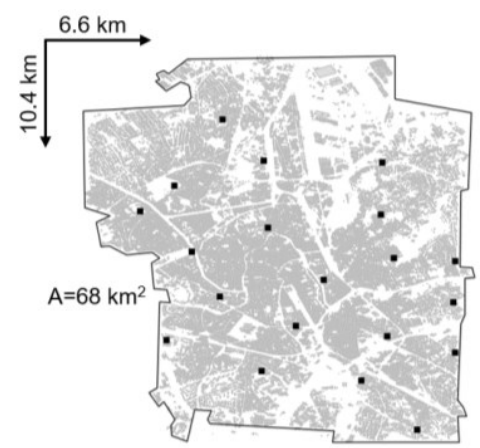

Fig. 1 Ghent City evaluation scenario.

We consider 224 simultaneous user connections. Some users require $1 \mathrm{Mbps}$ (data traffic) and other users $64 \mathrm{kbps}$ (voice traffic) [12]. The users are randomly distributed in the target area, with a 0.9 probability of data users and 0.1 of voice users. 
Each pixel in the map has the same probability to receive a user [13]. The traffic parameters are based on real data traffic from a local service provider.

We consider an initial setup based on a fixed outdoor over-roof antenna and Single-Input Single-Output (SISO) antenna configuration. The end-point connection at the user's home is provided by a transceiver to a Local Area Network (LAN). Fig. 2 shows the considered configuration, indicating that penetration losses [14] do not have to be accounted for the network planning.

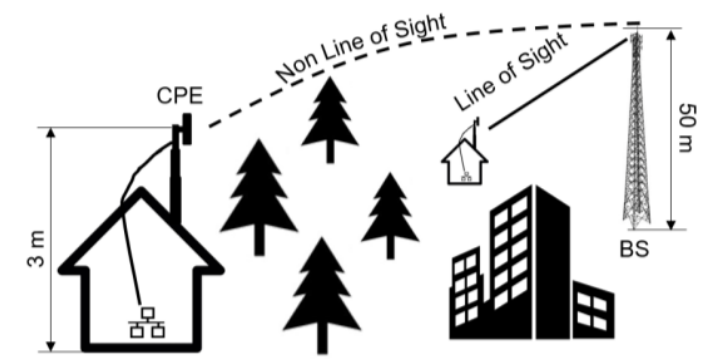

Fig. 2 Configuration for evaluation of TVWS technology.

We investigate the coverage, capacity and energy efficiency for IEEE 802.22 and IEEE 802.22b. The maximum allowable path loss is calculated based on the link budget parameters listed in Table I. These parameters are retrieved from specifications provided in the standards [6], [7], as well as values from manufacturers or published research [15], [16], [17], [18].

\section{TABLE I. LINK BUDGET PARAMETERS}

\begin{tabular}{|c|c|c|c|}
\hline Parameter & IEEE 802.22 & IEEE 802.22b & Unit \\
\hline Radiated power & 36 & 36 & $\mathrm{dBm}$ \\
\hline Frequency & 602 & 602 & $\mathrm{MHz}$ \\
\hline Bandwidth & 8 & 8 & $\mathrm{MHz}$ \\
\hline $\begin{array}{c}\text { Available TVWS } \\
\text { Channels }\end{array}$ & 25 & 25 & - \\
\hline $\begin{array}{c}\text { Cell interference } \\
\text { margin }\end{array}$ & 0 & 0 & $\mathrm{~dB}$ \\
\hline MIMO Gain & 0 & 0 & $\mathrm{~dB}$ \\
\hline $\begin{array}{l}\text { Total number of } \\
\text { subcarriers }\end{array}$ & 2048 & 1024 & - \\
\hline $\begin{array}{l}\text { Number of used } \\
\text { subcarriers }\end{array}$ & 1680 & $\begin{array}{l}840 \text { for US } \\
832 \text { for DS }\end{array}$ & - \\
\hline $\begin{array}{c}\text { Sampling } \\
\text { Frequency Factor }\end{array}$ & 1.1420 & 0.9325 & - \\
\hline Shadow Margin & $7.72 / 7.91$ & $7.72 / 7.91$ & $\mathrm{~dB}$ \\
\hline Fade Margin & 7.37 & 7.37 & $\mathrm{~dB}$ \\
\hline $\begin{array}{c}\text { Receiver } \\
\text { antenna gain }\end{array}$ & 11.5 & 11.5 & $\mathrm{~dB}$ \\
\hline $\begin{array}{c}\text { Receiver } \\
\text { feeder losses }\end{array}$ & 0.04 & 0.04 & $\mathrm{~dB}$ \\
\hline $\begin{array}{c}\text { Receiver } \\
\text { antenna height }\end{array}$ & 3 & 3 & $\mathrm{~m}$ \\
\hline $\begin{array}{c}\text { Base Station } \\
\text { antenna height }\end{array}$ & 50 & 50 & $\mathrm{~m}$ \\
\hline Noise Figure & 4 & 4 & $\mathrm{~dB}$ \\
\hline
\end{tabular}

The cell interference margin is considered $0 \mathrm{~dB}$. The reason is there are 25 non-interfering channels in Ghent [19] and we consider the strictest spectrum-sensing modes defined in the standards. The channel allocation for IEEE 802.22 and
IEEE $802.22 \mathrm{~b}$ is based in the detection of the wireless beacon (IEEE 802.22.1) with an occupancy decision threshold of $-116 \mathrm{dBm}$ [6], [7], [8]. We assume that a proper closed-loop geolocation database with similar constraint is in use to avoid interferences to/from primary broadcasting services [8]. For IEEE $802.22 \mathrm{~b}$ we considered the Orthogonal FrequencyDivision Multiplexing (OFDM) downstream (DS) configuration.

Table II lists the evaluated Modulation and Coding Schemes (MCS), its PHY bitrate and required Signal to Noise Ratio (SNR). The SNR recommended in standards guarantees a Bit Error Rate better than $10^{-7}$, considering the co-channel interference of a DVB-T2 TV broadcasting network [15].

TABLE II. EVALUATED MCS $\left(\right.$ BER $\left.<10^{-7}\right)$

\begin{tabular}{|c|c|c|c|}
\hline \multicolumn{2}{|c|}{ MCS (1/16 CP) } & $\begin{array}{c}\text { PHY } \\
\text { Bitrate }\end{array}$ & SNR \\
\hline $\mathbf{8 0 2 . 2 2}$ & $\mathbf{8 0 2 . 2 2 b}$ & Mbps & dB \\
\hline \multicolumn{2}{|c|}{ 1/2 QPSK } & 6.06 & 4.3 \\
\hline \multicolumn{2}{|c|}{ 1/2 16-QAM } & 12.09 & 10.2 \\
\hline 2/3 16-QAM & 16.12 & 12.4 \\
\hline 2/3 64-QAM & 24.18 & 18.3 \\
\hline 3/4 64-QAM & 27.21 & 19.7 \\
\hline- & 2/3 256-QAM & 32.24 & 26.9 \\
\hline- & 7/8 256-QAM & 42.32 & 28.2 \\
\hline
\end{tabular}

Fig. 3 shows two one-slope path loss models, Ghent City and Ghent Rural path loss model [20]. The coverage range is calculated by means of these models. Note that the Rural path loss model is only valid for receivers located in areas that satisfy the rural criteria defined in [20]. We consider $7.91 \mathrm{~dB}$ of shadow margin in the entire Ghent City scenario and $7.72 \mathrm{~dB}$ in the Rural scenario, for $90 \%$ of locations covered at a certain distance from the transmitter [20]. We consider $7.37 \mathrm{~dB}$ of fading margin for $99 \%$ of availability [17].

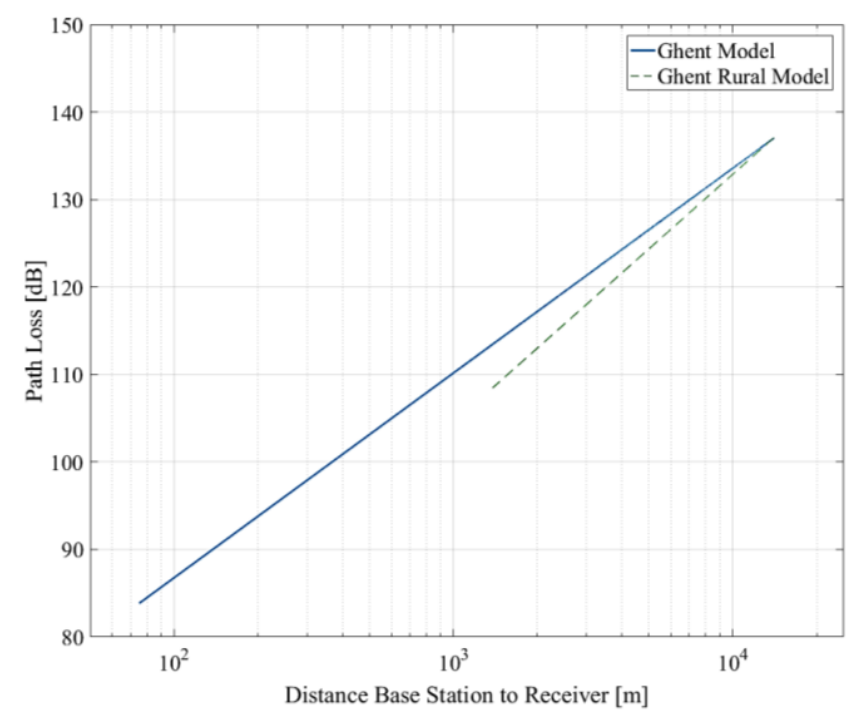

Fig. 3 Ghent City one-slope path loss model.

The total number of BSs depends on the number of BSs required to cover the target area and the number of BSs required to satisfy the traffic density. Equations 1 and 2 represent the minimum required number of BS taking into consideration the 
area constraint $\boldsymbol{N}_{\boldsymbol{B S a} \boldsymbol{a}}$ and traffic density constraint $\boldsymbol{N}_{\boldsymbol{B S} \boldsymbol{t}}$, respectively.

$$
N_{B S a}=\left\lceil\frac{A_{T}}{\pi \cdot R^{2}}\right\rceil
$$

where $\boldsymbol{A} \boldsymbol{T}\left(\mathrm{km}^{2}\right)$ is the target area and $\boldsymbol{R}(\mathrm{km})$ is the coverage range of the BS, with $\lceil\cdot\rceil$ the ceil function.

$$
N_{B S t}=\left\lceil\frac{T}{B_{B S}}\right\rceil
$$

where $\boldsymbol{T}$ (Mbps) is the maximal traffic demand of all simultaneous users within $\boldsymbol{A}_{\boldsymbol{T}}$, and $\boldsymbol{B}_{\boldsymbol{B} S}$ (Mbps) is the BS capacity. The actual number of required BSs will always be higher than $\boldsymbol{N}_{\boldsymbol{B S a}}$ and $\boldsymbol{N}_{\boldsymbol{B S t}}$ due to the deviation caused by the area geometry and BS location distribution.

In [21], a network simulation software to model and to optimize the power consumption of a wireless network in a realistic area is presented. By means of this software, a network planning simulation to account for the coverage and power consumption is performed for 30 different traffic patterns and user distributions. Each simulation represents an elapsed time interval $\boldsymbol{t}$ of one minute. Finally, the average energy efficiency of the BSs is calculated over these 30 simulations. A metric to account for the energy efficiency of a single BS is defined in [22]. By means of the Equation 3 is possible to account the TVWS BS energy efficiency $\boldsymbol{E} \boldsymbol{E}\left(\mathrm{km}^{2} \cdot \mathrm{Mbps} / \mathrm{W}\right)$.

$$
E E=\frac{\pi \cdot R^{2} \cdot u \cdot B}{P}
$$

where $\boldsymbol{R}$ is the coverage range of the BS, $\boldsymbol{u}$ is the number of users served by the BS, $\boldsymbol{B}$ is the total bitrate provided by the BS to these users, and $\boldsymbol{P}$ represents the power consumption of the BS. These parameters are retrieved from the network planning software for each BS at each time slot $t$. A progressive average is calculated to validate that the number of simulations are enough to guarantee a proper estimation of the mean percentage of users covered and the BS energy efficiency. For a reliable and fair comparison, the networks have to achieve a similar percentage of users covered.

We consider the power-consuming components of an outdoor BS and one single transmitter and sector per BS. As Fig. 4 shows the power-consuming components of an outdoor IEEE 802.22(b) BS are the Radio Unit (28W) [16], Power over Ethernet (4W) [23], Outdoor Optical Backhaul (32W) [24]. The considered power consumptions are referred to the BS peak time load traffic to investigate the worst-case scenario. In a scenario with an outdoor BS, the power consumption for the maximum radiated power is as low as $64 \mathrm{~W}$.

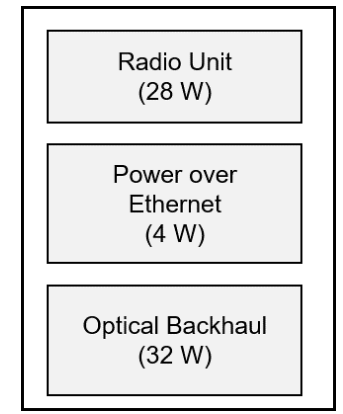

Fig. 4. IEEE 802.22(b) BS power-consuming components.

\section{RESULTS}

First, we calculate the coverage range. Fig. 5 shows the BS bitrate versus coverage range for entire Ghent and the rural environment. The IEEE 802.22 BS coverage range, considering Ghent model and bitrates from $6.06 \mathrm{Mbps}$ (1/2 QPSK) to $27.21 \mathrm{Mbps}$ (3/4 64-QAM), vary from $1.4 \mathrm{~km}$ to $6.4 \mathrm{~km}$. For the Rural model, the coverage range varies from $2.1 \mathrm{~km}$ to $7.5 \mathrm{~km}$. The coverage range for the rural model is $15 \%$ to $35 \%$ higher. This is due to a lower path loss between the BS and the user locations. The coverage range for IEEE $802.22 \mathrm{~b}$ BSs is $7 \%$ to $10 \%$ higher than for IEEE 802.22 , due to an improvement in the ratio of OFDM used from total subcarriers and a better sampling frequency factor (see Table I). Note that we consider same receiver specifications for both technologies (see Table I).

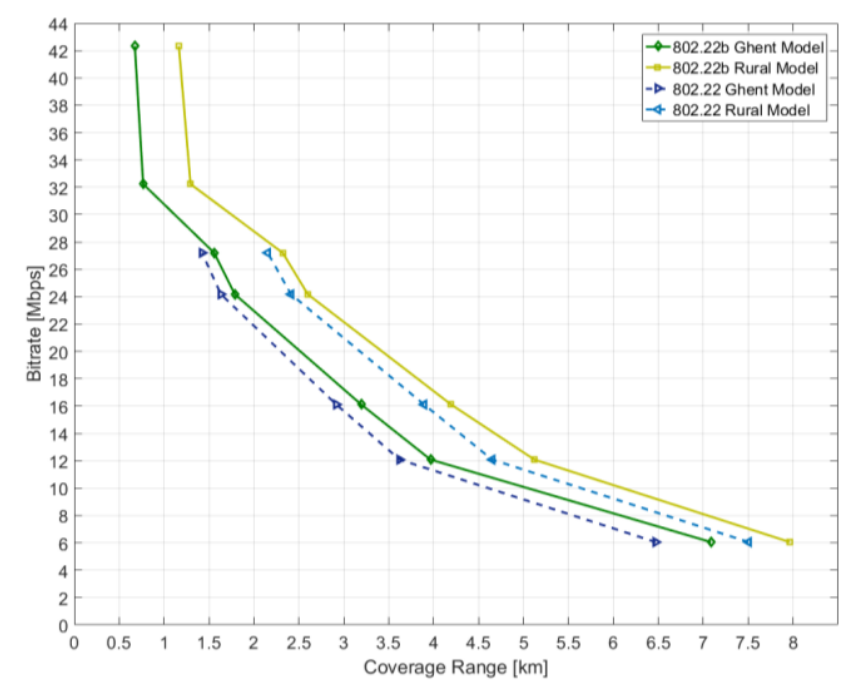

Fig. 5. IEEE 802.22 and IEEE $802.22 \mathrm{~b}$ bitrate versus coverage for the considered Ghent and Rural experimental path loss model.

Fig. 6 shows the minimum required number of BSs for each evaluated MCS, considering both experimental path loss models. The lowest number of required active BSs correspond to MCS 2/3 16-QAM, 2/3 64QAM and 3/4 64-QAM. This is because these MCSs achieve a better trade-off between area covered and capacity in the actual scenario. 


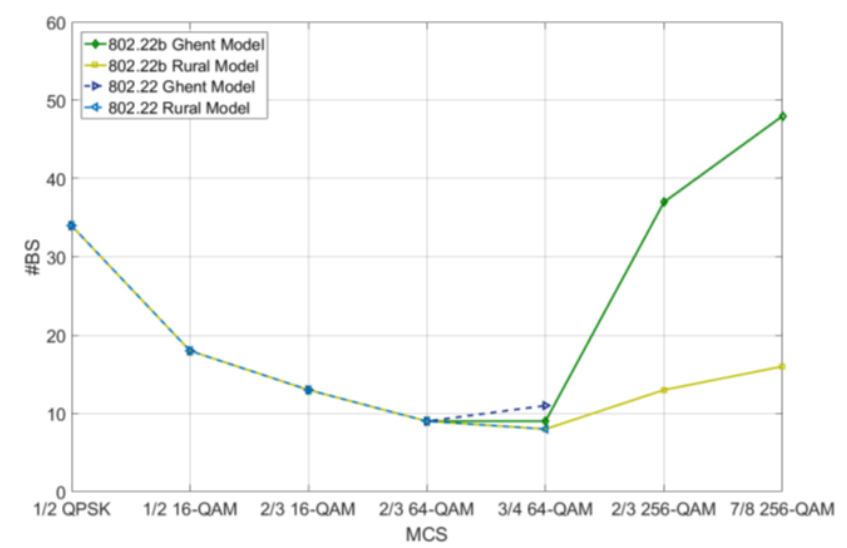

Fig. 6. Minimum required number of BS.

Fig. 7 shows the network coverage map. The percentage of users covered for IEEE $802.22 \mathrm{~b}$ is $98.3 \%$ to $99.8 \%$ and $97.4 \%$ to $99.2 \%$ for IEEE 802.22 , for Ghent model, and the Rural model, respectively. Note that the difference in the percentage of users covered is less than $2 \%$. The progressive average of the percentage of users covered, for 30 simulations is lower than $0.5 \%$. These conditions guarantee a reliable mean value and a fair comparison between both standards and experimental path loss models.

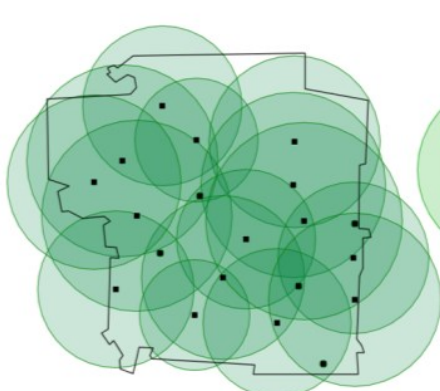

a)

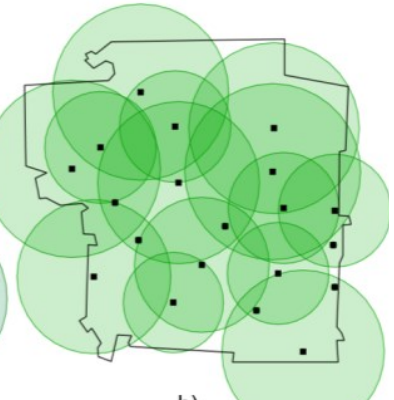

b)
Fig. 7. Network coverage maps for, a) IEEE 802.22b, b) IEEE 802.22.

The best percentage of coverage is achieved for the MCS $2 / 3$ 16-QAM, even when a slightly higher number of BSs is required in comparison with 2/3 64-QAM and 3/4 64-QAM. When considering only the BS coverage the minimum number of active BSs for 2/3 16-QAM is 2 to 3 and 5 to 11 for $3 / 4$ 64-QAM. The BS coverage will prevail in Ghent City scenario for MCSs with a similar minimum required number of $\mathrm{BS}$. This is due to the deviation caused by the area geometry and BS location distribution.

For each time slot $\mathbf{t}$ the network requires up to 15 different UHF channels to guarantee the non-interfering conditions described in Section II. Note that in a suburban scenario with similar traffic density but lower non-interfering channel availability, the detection occupancy threshold can be set as high as $-93 \mathrm{dBm}$ [25]. We extrapolated such condition to the Ghent scenario and the simulations reveal that the percentage of users covered is reduced to less than $60 \%$ for IEEE $802.22 \mathrm{~b}$. This is because a $12 \mathrm{~dB}$ cell inference margin has to be considered.
Fig. 8 shows the average energy efficiency of the BSs over time, for both technologies in the rural and suburban environment.

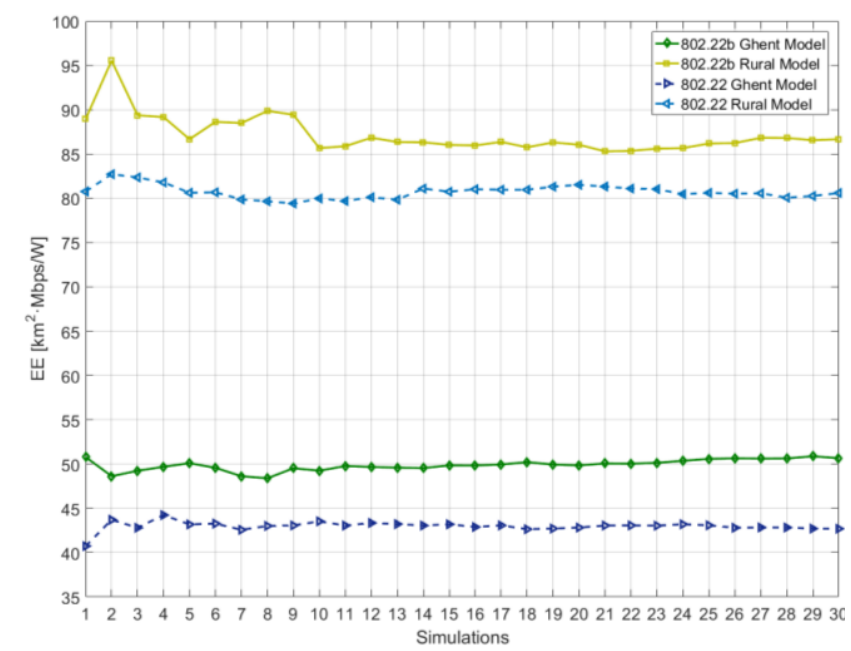

Fig. 8. IEEE 802.22 and IEEE 802.22b network energy efficiency comparison for the Ghent and the Rural experimental path loss models (progressive average).

IEEE $802.22 \mathrm{~b}$ BSs have an energy efficiency $7 \%$ to $15 \%$ higher than IEEE 802.22. This is because a higher coverage range per bitrate unit is achieved (see Fig. 4). For the Rural experimental model the BSs energy efficiency is $42 \%$ to $47 \%$ higher due to a lower path loss from the BS to the receiver locations (better propagation environment). After 12 simulations the standard deviation is lower than $2 \%$ for both standards and experimental path loss models.

\section{CONCLUSIONS}

In this paper we investigated the coverage, capacity and energy efficiency of IEEE 802.22 and IEEE 802.22b for the Ghent scenario for two experimental path loss models. We consider an outdoor scenario with fixed reception and a bitrate per data user of $1 \mathrm{Mbps}$ (around $90 \%$ of users).

For the Rural path loss model, the maximum coverage range is $7.9 \mathrm{~km}$ (IEEE 802.22b MCS 1/2 QPSK). For the entire Ghent propagation model, the maximum coverage range is $7.0 \mathrm{~km}$ (IEEE $802.22 \mathrm{~b}$ and $1 / 2$ QPSK). The highest energy efficiency is achieved for the MCS 2/3 16-QAM. The highest MCS (256-QAM modes) introduced in IEEE 802.22b does not lead towards a higher energy efficiency in Ghent scenario.

Future research will consist of the design of a power consumption model for current and future TVWS technologies. In addition, a comparison frame with optimized IEEE 802.11 af and LTE networks will be investigated.

\section{ACKNOWLEDGMENT}

Rodney Martinez Alonso is supported by LACETEL and a doctoral grant from the Special Research Fund (BOF) of Ghent University, Belgium. Margot Deruyck is a Post-Doctoral Fellow of the FWO-V (Research Foundation - Flanders, Belgium). 


\section{REFERENCES}

[1] Shared Spectrum Company, "General Survey of Radio Frequency Bands - $30 \mathrm{MHz}$ to $3 \mathrm{GHz}$," Vienna, Virginia, 2010.

[2] V. Valenta, R. Marsalek, G. Baudoin and M. Villegas, "Survey on Spectrum Utilization in Europe: Measurements, Analyses and Observations," 5th International ICST Conference on Cognitive Radio Oriented Wireless Networks and Communications, 2010.

[3] G. Guillen Nieto, "Digital TV: Oportunities and conectivity alternatives - Radio Cognitive," in 4th International Digital TV Forum, Havana, 2016.

[4] M. Murroni and e. al., "IEEE 1900.6: spectrum sensing interfaces and data structures for dynamic spectrum access and other advanced radio communication systems standard: technical aspects and future outlook," IEEE Communications Magazine, vol. 49, no. 12, pp. 118-127, 2011.

[5] M. Fadda, M. Murroni, C. Perra and V. Popescu, "TV white spaces exploitation for multimedia signal distribution," Signal Process. Image Communication, vol. 27, no. 8, pp. 893-899, 2012.

[6] IEEE Computer Society, "IEEE 802.22. Part 22: Cognitive Wireless RAN Medium Access Control (MAC) and Physical Layer (PHY) specifications: Policies and procedures for operation," IEEE, New York, 2011.

[7] IEEE Computer Society, "IEEE 802.22b. Part 22: Cognitive Wireless RAN Medium Access Control (MAC) and Physical Layer (PHY) Specifications: Policies and Procedures for Operation in the TV Bands," IEEE, New York, 2015.

[8] V. Popescu, M. Fadda, M. Murroni, J. Morgade and P. Angueira, "CoChannel and Adjacent Channel Interference and Protection Issues for DVB-T2 and IEEE 802.22 WRAN Operation," IEEE TRANSACTIONS ON BROADCASTING, vol. 60, no. 4, pp. 693-700, 2014.

[9] Dynamic Spectrum Alliance, "Worldwide Commercial Deployments, Pilots, and Trials," Dynamic Spectrum Alliance Limited, England, UK, 2016.

[10] A. B. Flores, R. E. Guerra, E. W. Knightly, P. Ecclesine and S. Pandey, "IEEE 802.11af: A Standard for TV White Space Spectrum Sharing," IEEE Communications Magazine, vol. 51, no. 10, pp. 92-100, 2013.

[11] K. Ishizu, K. Hasegawa, K. Mizutani, H. Sawada and e. al., "Field Experiment of Long-distance Broadband Communications in TV White Space Using IEEE 802.22 and IEEE 802.11af," WPMC2014, pp. 468473, 2014.

[12] M. Deruyck, J. Wyckmans, L. Martens and W. Joseph, "Emergency AdHoc Networks by Using Drone Mounted Base Stations for a Disaster Scenario," 16 IEEE 12th International Conference on Wireless and Mobile Computing, Networking and Communications (WiMob), pp. 1-7, 2016.
[13] M. Deruyck, W. Joseph, E. Tanghe and L. Martens, "Reducing the power consumption in LTE-Advanced wireless access networks by a capacity based deployment tool," Radio Science, no. 49, pp. 777-787, 2014.

[14] D. Plets, W. Joseph, L. Verloock, L. Martens, H. Gauderis and E. Deventer, "Extensive penetration loss measurements and models for different building types for DVB-H in the UHF band," IEEE TRANSACTIONS ON BROADCASTING, vol. 55, no. 2, pp. 213-222, 2009.

[15] V. Popescu, M. Fadda, M. Murroni and D. Giusto, "Coexistence Issues for IEEE 802.22 WRAN and DVB-T2 Networks," IEEE BMSB, 2016.

[16] M. Zennaro, E. Pietrosemoli and A. Sathiaseelan, "Architecting a Low Cost Television White Space Network For Developing Regions," Fifth ACM Symposium on Computing for Development, pp. 113-114, 2014.

[17] P. Angueira, M. Velez, D. d. 1. Vega, A. Arrinda and J. L. Ordiales, "Fading Caused by Moving Vehicles near the Receiver on DTV (COFDM) 8 MHz Signals," IEEE Communications Letters, vol. 6, no. 6, pp. 250-252, 2002.

[18] E. Klumperink, "Cognitive Radio CMOS Front-ends," University of Twente, Enschede, Netherlands, 2012.

[19] P. Piotrowski, "DVB-T Transmitters in Belgium," SAT Broadcast, [Online]. Available: http://www.satbroadcasts.com/DVBT_transmitters_in_Belgium.html. [Accessed 20 January 2017].

[20] D. Plets, W. Joseph, E. Tanghe, L. Verloock and L. Martens, "Analysis of propagation of actual DVB-H signal in a suburban environment," IEEE Antennas-and-Propagation-Society International Symposium, Vols. 1-12, pp. 1839-1842, 2007.

[21] M. Deruyck, E. Tanghe, W. Joseph and L. Martens, "Modelling and Optimization of power consumption in wireless access networks," Computer Communications, vol. 24, pp. 2036-2046, 2011.

[22] M. Deruyck, W. Joseph, B. Lannoo, D. Colle and L. Martens, "Designing Energy-Efficient Wireless Access Networks: LTE and LTE-Advanced," IEEE Internet Computing, vol. 17, no. 5, pp. 39-45, 2013.

[23] PLANET Technology Corporation, "IGS-624HPT: Industrial 4-Port 10/100/1000T 802.3at PoE," Taiwan, 2013.

[24] B. Mikkelsen, "Challenges and key technologies for coherent metro 100G transceivers," Light Waves, 2012. [Online]. Available: http://www.lightwaveonline.com/articles/print/volume-29/issue6/feature/challenges-and-key-technologies-for-coherent-metro-100gtransceivers.html.

[25] J. van de Beek, J. Riihijarvi and A. Achtzehn, "TV White Space in Europe," IEEE TRANSACTIONS ON MOBILE COMPUTING, vol. 11, no. 2 , pp. $178-188,2011$. 